\title{
Application of nano-carbon and titanium clip combined labeling in robot-assisted laparoscopic transverse colon cancer surgery
}

\author{
Nan Lin ${ }^{1 \dagger}$, Jiandong Qiu${ }^{2 \dagger}$, Junchuan Song ${ }^{3}$, Changwei Yu ${ }^{4}$, Yongchao Fang ${ }^{3}$, Weihang Wu ${ }^{1}$, Weijin Yang ${ }^{1}$ and
} Yu Wang ${ }^{1 *}$ (D)

\begin{abstract}
Background: Robot-assisted laparoscopic transverse colon tumor surgery requires precise tumor localization. The purpose of this study was to evaluate the safety and efficacy of nano-carbon and titanium clip combination labeling methods in robot-assisted transverse colon tumor surgery.

Methods: From January 2018 to January 2019, the clinical data of 16 patients who come from FuZhou, China underwent preoperative nano-carbon and titanium clip combined with robot-assisted laparoscopic transverse colon cancer surgery were retrospectively analyzed.

Results: Of the 16 patients, no signs of abdominal pain, fever, or diarrhea were observed after colonoscopy. Two titanium clips were seen on all of the 16 patients' abdominal plain films. Nano-carbon staining sites were observed during the operation, and no staining disappeared or abdominal cavity contamination. All patients underwent $\mathrm{R}_{0}$ resection. The average number of lymph nodes harvsted was $18.23 \pm 5.04$ (range, 9-32). The average time to locate the lesion under the laparoscopic was $3.03 \pm 1.26 \mathrm{~min}$ (range, 1-6 min), and the average operation time was $321.43 \pm 49.23 \mathrm{~min}$ (range, 240-400 min). All were consistent with the surgical plan, and there was no intraoperative change of surgical procedure or conversion to open surgery.

Conclusion: Preoperative colonoscopy combined with nano-carbon and titanium clip is safe and effective in robotassisted transverse colon cancer surgery. A At the same time, the labeling method shows potential in shortening the operation time, ensuring sufficient safety margin and reducing complications.
\end{abstract}

Keywords: Transverse colon cancer, Robot-assisted surgery, Colonoscopy, Nano-carbon

\section{Background}

Colorectal cancer is the third most commonly diagnosed cancer in males and is the second most commonly diagnosed cancer in females [1]. In recent years, with the improvement of people's living standards and dietary habits, the incidence of colon cancer is rising [2].

*Correspondence: flyfishwang@hotmail.com

${ }^{\dagger}$ Nan Lin and Jiandong Qiu contributed equally to this work

1 Department of General Surgery, 900 Hospital of the Joint Logistics Team,

Fuzhou, China

Full list of author information is available at the end of the article
Radical resection is the only cure treament for local colon cancer. In the past few decades, with the application of laparoscopy, the colorectal surgery has undergone significant changes. Compared with traditional open surgery, laparoscopic techniques allow improved visualization of areas difficult to reach by means of open surgery, and thus more precise dissection of anatomic structures [3], besides, laparoscopic surgery offers many advantages, such as minimizing surgical trauma, reducing blood loss, reducing postoperative pain, and promoting recovery [46]. Although laparoscopic surgery has many advantages, 
it still has some visual and operational limitations. Da Vinci robotic assisted surgery is an emerging minimally invasive technique that increases flexibility, improves surgical field of vision, and achieves optimal ergonomics [3]. However, for robotic surgery, accurate tumor localization is the key to the success of robotic colon surgery due to the lack of effective tactile feedback assistance, especially when the tumor locates in the transverse colon.

Currently, many methods are used for preoperative localization of colon tumors, including double-contrast barium enema, computed tomography colonography, titanium clip positioning, intraoperative colonoscopy, and preoperative injection stain positioning. Common staining agents for dyeing include methylene blue, indigo carmine, phthalocyanine green and Indian ink [7-10]. However, the above methods have their own shortcomings and limitations, such as radioactivity, inaccurate positioning, easy removal of titanium clips, and dispersion of stains [10-12]. In recent years, with the development of nanotechnology, carbon nanoparticles have been applied to tumor markers, such as colorectal cancer [13], breast cancer [14]. The injected carbon nanoparticle suspension contains nanometer carbon particles with an average diameter of $150 \mathrm{~nm}$. Due to molecular size and permeability, this ensures that these particles do not enter the blood circulation and have no toxic side effects on human body. Since 2007, China Food and Drug Administration approved the use of nano-carbon suspension in human.

In this study, we use nano-carbon and titanium clip combined labeling method to locate transverse colon tumor. Then, we performed robotic-assisted transverse colon tumor surgery and aimed to evaluate the safety and effectiveness of the approach in robotic-assisted surgery.

\section{Methods}

\section{Patients}

We retrospectively assessed 16 patients who were candidates for robot-assisted radical resection of transverse colon cancer at the General Surgery of the 900 Hospital of the Joint Logistics Support Force (FuZhou,China) from January 2018 to January 2019. Among them, 5 patients were female and 11 patients were male. All patiens were met the following inclusion criteria, including age of $18-70$ years old,had positive colonoscopic results for single transverse colon cancer,were stage I -III according to the TNM, no distant metastasis and no history of abdominal operations. Exclusion criteria included the following: patients before preoperative neoadjuvant chemoradiotherapy, benign tumors, had distant metastasis and patients who underwent emergency surgery, due to obstruction or perforation of the bowel. The surgeries were performed by the same team of surgeons. All surgical methods performed in this study were in accordance with the colorectal cancer guidelines of the National Comprehensive Cancer Network (NCCN). The tumornode-metastasis (TNM) staging was based on the seventh edition of the American Joint Committee on Cancer staging classification.

This study was approved by the Ethics Committee of the 900 Hospital of the Joint Logistics Support Force. Need for written informed consent was waived owing to the retrospective nature of the study.

\section{Materials}

Carbon nacoparticles suspension injection (Canaline) with a diameter of $150 \mathrm{~nm}$ was purchased from Chongqing LUMMY Pharmaceutical Co, Ltd.(Chongqing, China). Disposable injection needle (NM-200U-0423, Olympus, Japan), Rotary Titanium Clip Pusher ( HX5QR-1, Olympus, Japan) and Metal Titanium Clips (EZ Clip, HX-610-135 L, Olympus, Japan).

\section{Surgical procedure}

All patients underwent a standard mechanical bowel preparation the day before surgery. A team of 2 experienced endoscopists performed all colonoscopy procedures in the endoscopy centres affiliated with the 900 Hospital of the Joint Logistics Support Force. After a disposable injection needle (NM-200U-0423, Olympus, Japan) was used to inject $1 \mathrm{ml}$ saline into the submucosa under colonoscopy, a suitable submucosal apophysis was created. $0.1 \mathrm{ml}$ of nano-carbon was injected into the submucosal apophysis using another $1-\mathrm{ml}$ syringe. The nano-carbon syringe was removed and replaced with a first syringe containing saline, and the pinhole was washed with $1 \mathrm{~mL}$ of saline to flush the nano-carbon remaining (Fig. 1).

The nano-carbon is injected at an angle of $90^{\circ}$ and at a distance of $1 \mathrm{~cm}$ from the edge of the tumor [15]. Two titanium clips (EZ Clip, HX-610-135 L, Olympus, Japan) were individually placed anal-side and oral-side of the tumor from the edge of the tumor. Immediately after titanium clips placed, a plain abdominal X-ray was taken to confirm the position of the titanium clip (Fig. 2). All patients underwent pre-operative positioning 1-7 days before surgery.

\section{Results}

A total of 16 patients were included in the study, including $11 \mathrm{men}(68.8 \%)$ and 5 women $(31.2 \%)$, with an average age of $59.13 \pm 7.42$ years. Patient characteristics and tumor characteristics are shown in Table 1. No patients were found to have abdominal discomfort, fever, diarrhea, etc. after receiving colonoscopy. Two titanium clips were seen on all of the 16 patients' abdominal plain films. 


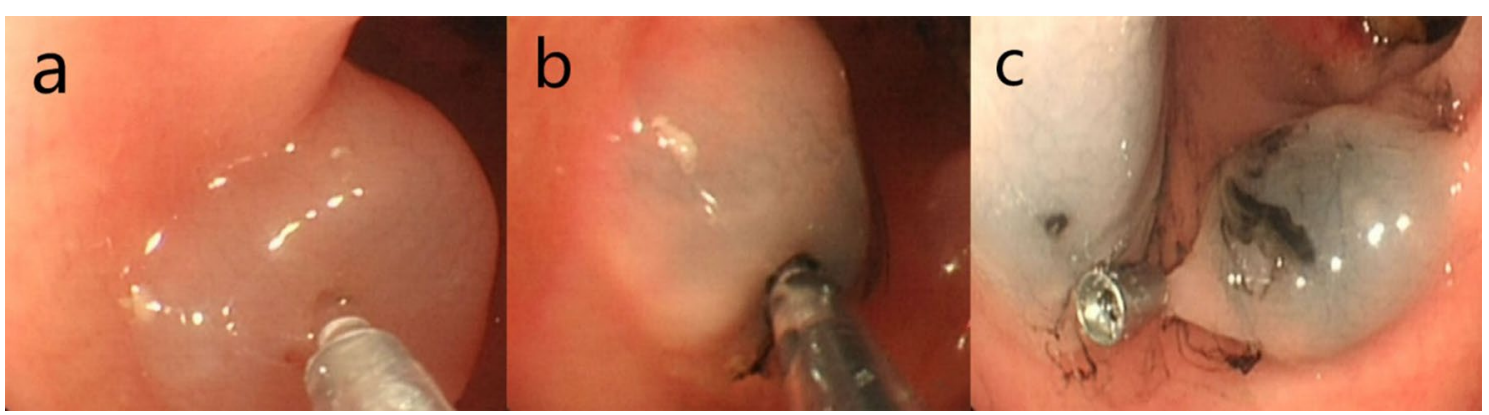

Fig. 1 Nano-carbon and titanium clip combination labeling methods under colonoscopy. a Injection $1 \mathrm{ml}$ of physiological saline into the submucosa layer to form a suitable submucosal apophysis. b Injection of $0.1 \mathrm{ml}$ nanocarbon into the submucosal apophysis. c Titanium clip placement

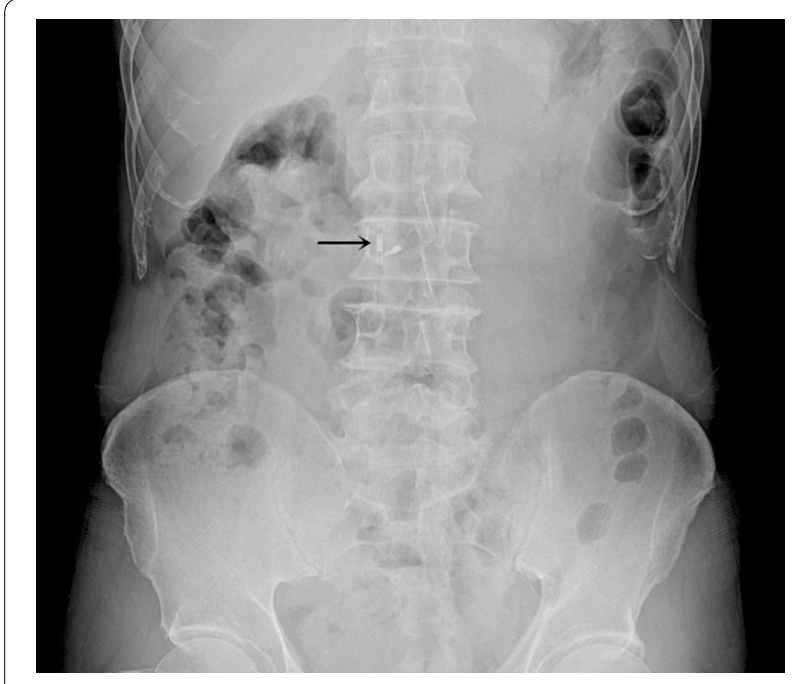

Fig. 2 Two titanium clips (black arrow) visible in the flat piece of the abdomen

All patients underwent robot-assisted surgery, including $5(31.25 \%)$ transverse colon resection, 3 (18.75\%) right colectomy, and 8 (50.00\%) left colectomy, as shown in Table 2. Nano-carbon staining sites were observed during the operation, and no staining disappeared or abdominal cavity contamination (Fig. 3). All patients in this study underwent $R_{0}$ resection. The number of lymph nodes harvsted in 16 patients was $18.23 \pm 5.04$ (range, 9-32). The average time to locate the lesion under the laparoscopic was $3.03 \pm 1.26 \mathrm{~min}$ (range, 1-6 $\mathrm{min}$ ), and the average operation time was $321.43 \pm 49.23 \mathrm{~min}$ (range, 240-400 min). All were consistent with the preoperative surgical plan, there was no intraoperative change of surgical procedure or conversion to open surgery. One patient developed postoperative intestinal obstruction and was discharged
Table 1 Patient characteristics and tumor characteristics

\begin{tabular}{ll}
\hline Variables & No. of patients (\%) \\
\hline Gender & \\
Male & $11(68.75 \%)$ \\
Female & $5(31.25 \%)$ \\
Mean age (years) & $59.13 \pm 7.42$ \\
Tumor staging(TNM) & \\
Tstage & \\
Tis & $1(6.25 \%)$ \\
T1 & $3(18.75 \%)$ \\
T2 & $6(37.50 \%)$ \\
T3 & $4(25.00 \%)$ \\
T4 & $2(12.50 \%)$ \\
N stage & \\
N0 & $9(56.25 \%)$ \\
N1 & $6(37.50 \%)$ \\
N2 & $1(6.25 \%)$ \\
\hline
\end{tabular}

successfully after conservative treatment for 12 days. One patient developed pulmonary infection due to her advanced age and late post-operative activities.

Postoperative pathology confirmed that the surgical margins were negative, and no residual carbon particles were observed. The average length of the proximal margin was $6.40 \pm 3.29 \mathrm{~cm}$ (range, $3.9-18.0 \mathrm{~cm}$ ), and the distal margin was $9.97 \pm 3.89 \mathrm{~cm}$ (range $5.5-21.0 \mathrm{~cm}$ ).

\section{Discussion}

In 2002, Hashizume and Weber et al. first reported robotic colectomy $[16,16]$. Since then, more and more studies have shown that robotic colorectal surgery has similar oncological results compared with laparoscopic or open surgery $[18,18]$, the application of robotic surgery in the field of general surgery has increased year by year. Although laparoscopy has now become the gold 
Table 2 Clinical results of nano-carbon and titanium clip markers

\begin{tabular}{|c|c|}
\hline Variables & No. of patients (\%) \\
\hline \multicolumn{2}{|l|}{ Surgical approach } \\
\hline $\begin{array}{l}\text { Transverse colec- } \\
\text { tomy }\end{array}$ & $5(31.25 \%)$ \\
\hline $\begin{array}{l}\text { Right hemicolec- } \\
\text { tomy }\end{array}$ & $3(18.75 \%)$ \\
\hline Left hemicolectomy & $8(50.00 \%)$ \\
\hline \multicolumn{2}{|c|}{ Tumor localization by nano-carbon } \\
\hline Precise & 16 \\
\hline Dyeing dispersion & 0 \\
\hline Dyeing disappears & 0 \\
\hline \multicolumn{2}{|c|}{ Postoperative complications } \\
\hline Bleeding & 0 \\
\hline Anastomotic fistula & 0 \\
\hline $\begin{array}{l}\text { Intestinal obstruc- } \\
\text { tion }\end{array}$ & 1 (6.75\%) \\
\hline Infection & $1(6.75 \%)$ \\
\hline
\end{tabular}

standard for a variety of relatively easy general surgery. However, laparoscopic ergonomics and technical limitations, the loss of anatomical orientation due to twodimensional views make the identification of important structures a problem. The extraordinary visual and ergonomic advantages of the Da Vinci system were presumed to overcome the limitations of laparoscopy and improve the results of minimally invasive colon surgery [20]. Although the application of the Da Vinci system in the general field has increased year by year, due to the lack of effective tactile feedback assistance, intraoperative exploration is often difficult when the tumor is small or does not invade the serosa. It has been reported that the wrong colon segment is removed during laparoscopic surgery, which requires conversion to open surgery and resection of longer intestine segments [21, 22].

For colon tumors, colonoscopy is still the most sensitive diagnostic tool, but due to the lack of obvious anatomical landmarks in the colon, inaccurate tumor localization may lead to longer lengths of resection, and even the removal of normal intestinal segments leaving the tumor. $16.7 \%$ of cases will have different procedures from the original plan due to inaccurate preoperative colonoscopy, especially for transverse colon tumors [23]. So the 2013 Society of American Gastrointestinal and Endoscopic Surgeons (SAGES) and the 2004 European Association of Endoscopic Surgery (EAES) clinical practice guidelines for laparoscopic resection of colon cancer recommend colonoscopic tattooing of small lesions [24, 25]. But until now there were no evidence that suggest that colonoscopic tattooing should be used only for small lesions. Moreover, tumors localized in the transverse

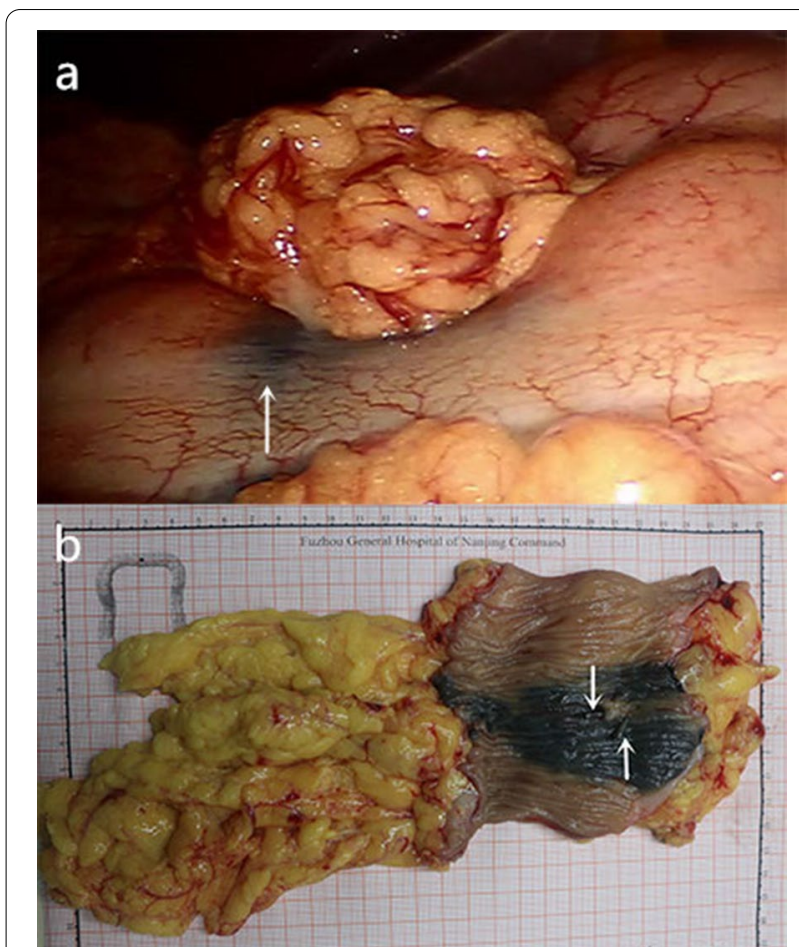

Fig. 3 a Serosal appearance of colonic lesion labeled by nano-carbon in robot-assisted surgery (The white arrow points to the nano-carbon black stained area). b Mucosal appearance of the surgical removal specimen, which can observe nano-carbon black stained area and two titanium clips (white arrow).

colon are commonly considered challenging, and routine tattooing of these tumors is recommended. Because the tumor is located close to the liver curvature, spleen curvature and the middle part of the transverse colon, the resection range is very different, so the surgical approach is divided into left hemicolectomy, right colon colectomy and transverse colon resection. In the Da Vinci surgical system, the three surgical methods, the placement of the robot and the port are different. If the surgical plan is changed during the operation, it will not only directly increase the cost of surgery, but also lead to prolonged operation time and increased risks of surgery. Therefore, how to achieve accurate preoperative and intraoperative positioning determines the premise of the success of robotic surgery.

There are a number of techniques currently used for the localization of colonic lesions, including double-contrast barium enema, computed tomography colonography, titanium clip positioning, intraoperative colonoscopy, and preoperative injection stain positioning. But each method has its shortcomings and limitations. Doublecontrast barium enema and computed tomography colonography are easy to miss smaller lesions $[12,26]$. 
Titanium clip positioning is a short-term solution, costly, often shifts or falls off after 2-3 weeks of implantation; and the titanium clip is small, the clip cannot be seen from the serosal side, and it cannot be touched during laparoscopic surgery [27]. Intraoperative colonoscopy can also be used for positioning, but it is a more complex method that requires experienced endoscopists and specific equipment in the operating room, which can increase the time of surgery and increase the risk of anesthesia and the probability of infection [7]. In addition, colonoscopy will inflate the intestines, increasing the difficulty of surgery [13]. In recent years, the most common method of positioning has been to inject a stain into the intestinal wall. Commonly used dyes such as methylene blue, indigo carmine, and phthalocyanine green have relatively short dyeing times, which tend to spread over time and contaminate the surgical field of view and cause inaccurate positioning $[28,29]$. Although Indian inks have a long time at the marked parts, some studys reported Indian ink can cause peritonitis, cellulitis, gastritis, colonic abscesses and inflammatory pseudotumors [30-32].

The nano-carbon used in this study, Askin M P et al., evaluated the safety and efficacy of colon labeling using nano-carbon in a study of 113 patients [33]. In the study, no patients developed fever, abdominal pain or symptoms of inflammation, and nano-carbons existed for 1 year, confirming that nano-carbon is a long-term safe and effective marker. In this study, we also did not find any discomfort after the patient received nano-carbon injection. In addition, we use the "four quadrant" method [15] (ie, four points are selected centered on the tumor, each point is $90^{\circ}$ apart) injection labeling is performed around the tumor to avoid staining of the intestinal wall when the tumor is on the mesenteric side. Moreover, the marker points are $1 \mathrm{~cm}$ away from the tumor and avoid direct injection into the tumor. Secondly, the needle is at an angle of $45^{\circ}$ to the wall of the intestine when the needle is inserted, because the vertical needle easily penetrates the intestinal wall, causing the dye to enter the mesentery or the abdominal cavity to contaminate the surgical field of view. The diffusion of nano-carbon can also be reduced by the "three-step injection method" of J. W. Park et al. [34]. The final intraoperative findings showed that all 16 patients were able to find nano-carbon labeled sites, confirming that our approach worked. In this study, we placed a titanium clip on each of the anal side and the mouth side, and then immediately examined the radiation. The titanium clip showed a high signal in the X-ray, and the tumor was located between the two titanium clips. Studies have shown that two titanium clips are used to prevent displacement or shedding when using titanium clips for colon marking [27]. In general, the peak period of shedding is $2-3$ weeks after placement, the longer the time, the greater the probability of shedding. Radiation inspection immediately after the titanium clip is placed can reduce errors caused by displacement or shedding of the titanium clip. Moreover, all patients underwent surgery within 1 week after receiving the marker, thereby avoiding the peak of titanium clip detachment.

Nano-carbon labeling helps us to quickly find tumors during surgery, avoid excision of the wrong bowel segment, and ensure a sufficient safety margin. Titanium clip marking allows us to obtain a more accurate positioning before surgery, which helps to develop a surgical plan and avoid the cost associated with robotic surgery and additional operative time due to changes to the surgical plan. Accurate preoperative positioning can provide a reliable basis for the selection of Trocar position and surgical incision for laparoscopic surgery, to avoid surgical errors due to poor exposure of the surgical field caused by incorrect selection of Trocar position. In general, robotic surgery has a longer operation time than laparoscopic surgery. Although long operation time may be related to high postoperative morbidity, operative time is not the only parameter showing the quality of surgery and it is obvious that the operation time may decrease as the experience of robotic surgery increases. And our method can significantly reduce the exploration time.

\section{Conclusion}

In conclusion, the findings of this study have shown that the preoperative colonoscopy nano-carbon and titanium clip combined labeling method is safe and effective in robot-assisted transverse colon cancer surgery. At the same time, the labeling method shows potential in shortening the operation time, ensuring sufficient safety margin and reducing complications.

\section{Acknowledgements \\ We thank all participants who contributed to the study.}

\section{Authors' contributions}

$\mathrm{NL}$, JDQ, and YW conceived and design the study. JDQ wrote the manuscript text. JCS, CWY, YCF collection and assembly of data. WHW, WJY data analysis and interpretation. NL, YW administrative and technical support. All authors read and approved the final manuscript.

\section{Funding}

This work was supported by a grant from Fujian Province Science and Technology Plan Project External Cooperation Project (No.201710101). The corresponding author is the first person in charge of the fund.

Availability of data and materials

The datasets used and analysed during the current study are available from the corresponding author on reasonable request. 


\section{Declarations}

\section{Ethics approval and consent to participate}

Ethical approval was given by the 900 Hospital of the Joint Logistics Team. Need for written informed consent was waived owing to the retrospective nature of the study.

\section{Consent for publication}

Not applicable.

\section{Competing interests}

The authors declared no potential conflicts of interest with respect to the research, authorship, and/or publication of this article.

\section{Author details}

1 Department of General Surgery, 900 Hospital of the Joint Logistics Team, Fuzhou, China. ${ }^{2}$ Department of Oncological Surgery, Sanming First Hospital Affiliated to Fujian Medical University, Fuzhou, China. ${ }^{3}$ Department of General Surgery, Dongfang Hospital, Xiamen University, Xiamen, China. ${ }^{4}$ Clinical Institute of Fuzhou General Hospital, Fujian Medical University, Fuzhou, China.

Received: 5 July 2020 Accepted: 13 May 2021

Published online: 24 May 2021

\section{References}

1. Ferlay J, Soerjomataram I, Dikshit R, Eser S, Mathers C, Rebelo M, Parkin DM, Forman D, Bray F. Cancer incidence and mortality worldwide: sources, methods and major patterns in globocan 2012. Int J Cancer. 2015;136:E359-86.

2. Chen $\mathrm{W}$, Zheng $\mathrm{R}$, Zeng $\mathrm{H}$, Zhang $\mathrm{S}$. The incidences and mortalities of major cancers in China 2010. Chin J Cancer. 2014;33:402-5.

3. Antoniou SA, Antoniou GA, Koch OO, Pointner R, Granderath FA. Robotassisted laparoscopic surgery of the colon and rectum. Surg Endosc 2012;26:1-11

4. Bartels SA, Vlug MS, Hollmann MW, Dijkgraaf MG, Ubbink DT, Cense HA van Wagensveld BA, Engel AF, Gerhards MF, Bemelman WA. Small bowe obstruction, incisional hernia and survival after laparoscopic and open colonic resection (LAFA study). Br J Surg. 2014;101:1153-9.

5. Bernasconi M, Metzger J. Randomized clinical trial comparing laparoscopic and open surgery in patients with rectal cancer. Br J Surg. 2010;97:619-20.

6. Kouhia ST, Heiskanen JT, Huttunen R, Ahtola HI, Kiviniemi VV, Hakala T. Long-term follow-up of a randomized clinical trial of open versus laparoscopic appendicectomy. Br J Surg. 2010;97:1395-400.

7. Zmora O, Dinnewitzer AJ, Pikarsky AJ, Efron JE, Weiss EG, Nogueras JJ. Intraoperative endoscopy in laparoscopic colectomy. Surg Endosc. 2002;16:808-11.

8. Halligan S, Wooldrage K, Dadswell E, Kralj-Hans I, von Wagner C, Edwards R, Yao G, Kay C, Burling D, Faiz O, Teare J, Lilford RJ, Morton D, Wardle J, Atkin W. Computed tomographic colonography versus barium enema for diagnosis of colorectal cancer or large polyps in symptomatic patients (SIGGAR): a multicentre randomised trial. The Lancet. 2013:381:1185-93.

9. Gorgun IE, Aytac E, Manilich E, Church JM, Remzi FH. Intraoperative colonoscopy does not worsen the outcomes of laparoscopic colorectal surgery: a case-matched study. Surg Endosc. 2013;27:3572-6.

10. Yeung JMC, Maxwell-Armstrong C, Acheson AG. Colonic tattooing in laparoscopic surgery_-making the mark? Colorectal Dis. 2010:11(5):527-30.

11. Yan J, Xue F, Chen H, Wu X, Zhang H, Chen G, Lu J, Cai L, Xiang G, Deng Z, Zheng Y, Zheng X, Li G. A multi-center study of using carbon nanoparticles to track lymph node metastasis in t1-2 colorectal cancer. Surg Endosc. 2014;28:3315-21.

12. Rockey DC, Paulson E, Niedzwiecki D, Davis W, Bosworth HB, Sanders L, Yee J, Henderson J, Hatten P, Burdick S, Sanyal A, Rubin DT, Sterling M, Akerkar G, Bhutani MS, Binmoeller K, Garvie J, Bini EJ, McQuaid K, Foster WL, Thompson WM, Dachman A, Halvorsen R. Analysis of air contrast barium enema, computed tomographic colonography, and colonoscopy: prospective comparison. Lancet. 2005;365:305-11.

13. Wang W, Wang R, Wang Y, Yu L, Li D, Huang S, Ma J, Lin N, Yang W, Chen $X$, Liu B, Lv R, Liao L. Preoperative colonic lesion localization with charcoal nanoparticle tattooing for laparoscopic colorectal surgery. J Biomed Nanotechnol. 2013:9:2123-5.

14. Jiang Y, Lin N, Huang S, Lin C, Jin N, Zhang Z, Ke J, Yu Y, Zhu J, Wang Y. Tracking nonpalpable breast cancer for breast-conserving surgery with carbon nanoparticles: implication in tumor location and lymph node dissection. Medicine. 2015:94:e605.

15. Hyman N, Waye JD. Endoscopic four quadrant tattoo for the identification of colonic lesions at surgery. Gastrointest Endosc. 1991;37:56-8.

16. Hashizume M, Shimada M, Tomikawa M, Ikeda Y, Takahashi I, Abe R, Koga F, Gotoh N, Konishi K, Maehara S, Sugimachi K. Early experiences of endoscopic procedures in general surgery assisted by a computer-enhanced surgical system. Surv Methodol. 2002;16:1187-91.

17. Weber PA, Merola S, Wasielewski A, Ballantyne GH. Telerobotic-assisted laparoscopic right and sigmoid colectomies for benign disease. Dis Colon Rectum. 2002:45:1689-94

18. Kim CW, Kim CH, Baik SH. Outcomes of robotic-assisted colorectal surgery compared with laparoscopic and open surgery: a systematic review. J Gastrointest Surg. 2014;18:816-30.

19. Lin S, Jiang HG, Chen ZH, Zhou SY, Liu XS, Yu JR. Meta-analysis of robotic and laparoscopic surgery for treatment of rectal cancer. World J Gastroenterol. 2011:17:5214-20.

20. Gorgun E, Aytac E, Gurland B, Costedio MM. Case-matched comparison of robotic versus laparoscopic colorectal surgery: initial institutional experience. Surg Laparosc Endosc Percutan Tech . 2015;25(5):e148-51.

21. Wishner JD, Baker JW, Hoffman GC, Hubbard GW, Gould RJ, Wohlgemuth SD, Ruffin WK, Melick CF. Laparoscopic-assisted colectomy_the learning curve. Surg Endosc. 1995;9(11):1179-83.

22. Wexner SD, Cohen SM, Ulrich A, Reissman P. Laparoscopic colorectal surgery - are we being honest with our patients? Dis Colon Rectum. 1995:38:723-7.

23. Fernandez LM, Ibrahim RNM, Mizrahi I, DaSilva G, Wexner SD. How accurate is preoperative colonoscopic localization of colonic neoplasia? Surg Endosc. 2019;33:1174-9.

24. Veldkamp R, Gholghesaei M, Bonjer HJ, Meijer DW, Buunen M, Jeekel J, Anderberg B, Cuesta MA, Cuschierl A, Fingerhut A, Fleshman JW, Guillou PJ, Haglind E, Himpens J, Jacobi CA, Jakimowicz JJ, Koeckerling F, Lacy AM, Lezoche E, Monson JR, Morino M, Neugebauer E, Wexner SD, Whelan $\mathrm{RL}$. Laparoscopic resection of colon cancer: consensus of the european association of endoscopic surgery (EAES). Surg Endosc. 2004;18:1163-85.

25. Zerey M, Hawver LM, Awad Z, Stefanidis D, Richardson W, Fanelli RD. Sages evidence-based guidelines for the laparoscopic resection of curable colon and rectal cancer. Surg Endosc. 2013;27:1-10.

26. Atkin W, Dadswell E, Wooldrage K, Kralj-Hans I, von Wagner C, Edwards R, Yao G, Kay C, Burling D, Faiz O, Teare J, Lilford RJ, Morton D, Wardle J, Halligan S. Computed tomographic colonography versus colonoscopy for investigation of patients with symptoms suggestive of colorectal cancer (SIGGAR): a multicentre randomised trial. The Lancet. 2013:381(9873):1194-202.

27. Ohdaira T, Konishi F, Nagai H, Kashiwagi H, Shito K, Togashi K, Kanazawa K. Intraoperative localization of colorectal tumors in the early stages using a marking clip detector system. Dis Colon Rectum. 1999;42(10):1353-5. 
28. Price N, Gottfried MR, Clary E, Lawson DC, Baillie J, Mergener K, Westcott C, Eubanks S, Pappas TN. Safety and efficacy of india ink and indocyanine green as colonic tattooing agents. Gastrointest Endosc. 2000;51(4):438-42.

29. Committee AT, Kethu SR, Banerjee S. Endoscopic tattooing. Gastrointest Endosc. 2010;72:681-5.

30. Hornig D, Kühn H, Stadelmann O, Bötticher R. Phlegmonous gastritis after India Ink marking. Endoscopy. 1983;15:266-9.

31. Park SI, Genta RS, Romeo DP, Weesner RE. Colonic abscess and focal peritonitis secondary to india ink tattooing of the colon. Gastrointest Endosc. 1991;37:68-71.

32. Coman E, Brandt LJ, Brenner S, Frank M, Sablay B, Bennett B. Fat necrosis and inflammatory pseudotumor due to endoscopic tattooing of the colon with india ink. Gastrointest Endosc. 1991;37:65-8.
33. Askin MP, Waye JD, Fiedler L, Harpaz N. Tattoo of colonic neoplasms in 113 patients with a new sterile carbon compound. Gastrointest Endosc 2002;56:339-42.

34. Park JW, Sohn DK, Hong CW, Han KS, Choi DH, Chang HJ, Lim SB, Choi HS, Jeong SY. The usefulness of preoperative colonoscopic tattooing using a saline test injection method with prepackaged sterile india ink for localization in laparoscopic colorectal surgery. Surg Endosc. 2008;22:501-5.

\section{Publisher's Note}

Springer Nature remains neutral with regard to jurisdictional claims in published maps and institutional affiliations.
Ready to submit your research? Choose BMC and benefit from:

- fast, convenient online submission

- thorough peer review by experienced researchers in your field

- rapid publication on acceptance

- support for research data, including large and complex data types

- gold Open Access which fosters wider collaboration and increased citations

- maximum visibility for your research: over $100 \mathrm{M}$ website views per year

At BMC, research is always in progress.

Learn more biomedcentral.com/submissions 